\title{
Gut Microbiota and its Implications on the Progression of Gastrointestinal Cancer
}

\author{
Bessi Qorri ${ }^{1,2}$, Manpreet Sambi1,2 and Myron R Szewczuk ${ }^{1 *}$ \\ ${ }^{1}$ Department of Biomedical and Molecular Sciences, Queen's University, Kingston, Canada \\ ${ }^{2}$ Contributing first authorship, Canada
}

Submission: August 11, 2017; Published: August 15, 2017

"Corresponding author: Myron R Szewczuk, Department of Biomedical and Molecular Sciences, Queen's University, Kingston, ON K7L 3N6, Canada, Tel: +1 6135332457; Fax: +16135336796; Email: szewczuk@queensu.ca

\begin{abstract}
The gastrointestinal (GI) tract is colonized by trillions of microorganisms that play a vital role in maintaining homeostasis, contributing to several local and systemic processes including digestion, and have been shown to influence the immune response. Although the crucial contributions of the gut microbiota have been studied in great detail in relation to normal GI function, recent studies have exposed a previously unknown link between commensal bacteria, cancer progression, and treatment efficacy. Preliminary studies have shown that an imbalance in gut microbiota may influence cancer progression in part due to the development of an inflammatory environment that potentiates cancer progression and the possibility of driver and passenger microorganisms that induce DNA damage. Furthermore, in relation to the efficacy of immunotherapies, several microorganisms are involved in enhancing the anti-tumor response by helping to activate tumor antigen specific T-cells. However, this particular area of research is in its infancy and requires additional studies to further understand the implications of the link between commensal bacteria and cancer progression.
\end{abstract}

Keywords: Microbiota; Gastrointestinal cancer; Colorectal cancer; Chemotherapy; Inflammation

\section{Introduction}

The gut microbiota (flora) consists of trillions of bacteria, viruses, and fungi that colonize the human intestine beginning at birth, and acts as a natural defensive barrier to infection and as an organ in influencing virtually every vital function $[1,2]$. The gut microbiota plays an essential structural role in the architecture of the intestinal epithelium as delineated from germ-free mice which demonstrated a lack of long intestinal villi, crypt atrophy, and slow epithelial cell renewal [3]. These structural intestinal epithelial modifications that take place are of utmost importance because individuals can become more susceptible to infection, ulcer development, irritable bowel syndrome [4], Crohn's disease [5], and cancer progression [6] if this microbiota barrier is compromised. Although the relationship between cancer progression and the microbiota is a rather recent advancement, it is perhaps not surprising because of the commensal role that gut bacteria plays in maintaining host health and its effects on the progression of other diseases. Here we will briefly introduce the relationship between the gut microbiota and the gastrointestinal (GI) tract and its link in colorectal cancer (CRC) progression through the regulatory effects of the gut microbiota and their role in enhancing or dampening the efficacy of anticancer therapies.

\section{The connection between gut flora and colorectal cancer progression}

The initiation of a cancerous phenotype is typically attributed to the genetic alteration of tumor suppressor genes and oncogenes that control the cell cycle; however, carcinogen exposure, environmental factors and hereditary predisposition $[7,8]$ are known to mediate cancer progression. It has also been well established that bacteria [9] and viruses [10] are capable of affecting cancer progression. However, more recent research has suggested that commensal bacteria in the gut microbiota that normally exert positive effects on digestive health may also be associated with mediating cancer progression [11].

The structural role of the gut microbiota on intestinal epithelium integrity is particularly relevant in CRC as this cancer progression is accompanied by severe physiological alterations [12].The gut microbiota also plays a metabolic role through the digestion, degradation, and production of short-chain fatty acids 
(SCFA) which regulate the permeability of the epithelial barrier [13]. The increased tight junction permeability associated with deregulated gut microbiota triggers an inflammatory cascade that is associated with obesity and insulin sensitivity through SCFA, and has implications on gut integrity, glucose homeostasis and lipid metabolism [1,13]. In addition, the increased permeability allows innate immune receptors such as Toll-like receptors (TLRs) and Nod-like receptors (NLRs), to recognize particular molecular motifs associated with pathogens. Once pathogens are detected, the resulting response involves the activation of several signaling pathways including MAPK, NF- $\varkappa$ $\mathrm{B}$, and PI3K/AKT which ultimately lead to an increase in proinflammatory cytokine expression and further contribute to the inflammatory microenvironment [14]. This inflammatory response has important implications, as it has been repeatedly associated with cancer initiation and progression.

Other theories suggest that there might be a direct association between bacteria and CRC such that they facilitate cancer progression by damaging DNA in the colorectal epithelium [15]. This model manipulates and applies the driver and passenger gene theory to specialized bacteria. As they apply to microbiota, driver microorganisms initiate DNA damage and passenger bacteria populate the tumor site and promote tumorigenesis [15]. Furthermore, candidate species of the gut microbiota have been suggested to play particularly significant roles in potentially enhancing the progression of CRCs and is discussed in detail by Gao et al. [16]. In brief, Fusobacterium nucletum a pathogenic microorganism that has not only been shown to enhance the pro-inflammatory microenvironment and thereby mediating the progression of CRC, but is also capable of inhibiting the action of natural killer (NK) cells that normally exhibit an anti-tumor response. Escherichia coli has also been shown to play a role in increasing the risk of CRC, but the mechanism(s) of action remains unclear. Nonetheless, it is capable of inducing DNA damage and gene mutations that can contribute to the progression of CRC. Bacteroides fragilis, on the other hand, has been shown to mediate DNA damage as well as activate proliferation pathways, and thus could be considered a candidate driver microorganism. Contradictory evidence, however, has shown that this particular species of bacteria is required to enhance immunostimulatory effects of immunotherapy involving CTLA-4 inhibition [17]. Another commensal bacteria, Bifidobacterium, has been implicated in mediating an antitumor immune response and enhancing the antitumor effects of immunotherapy involving the PD-L1 checkpoint blockade [18]. This enhanced immune response was evidenced by an increase in tumor-specific T-cells and an increase in CD8+ T-cells infiltrating the tumor. These effects were further enhanced when a live bacterial culture was administered, suggesting that these bacteria may colonize a region of the gut that allow it to interact with host cells to regulate the action of dendritic cells [18].

To this end, the commensal bacteria composition may play an important role in cancer progression; however, due to the limited information that is available that provides a direct causal relationship between the gut microbiota and CRC and more research is required in order to solidify this connection.

\section{Imbalances in gut microbiota and the implications on the efficacy of cancer therapy}

Imbalances in the gut microbiota have been implicated in both cancer progression and the efficacy of cancer therapy. Environmental factors such as antibiotics, xenobiotics and smoking, as well as pathogenic challenges, aging-related alterations, metabolic cues, and genetic factors contribute to the dysregulation of the gut microbiota. This disregulation is associated with hematopoietic dysfunction, metabolic alterations, infectious diseases, immunological defects and chronic inflammation, all of which are known to contribute to and ultimately promote oncogenesis [3]. The gut microbiota has been shown to not only regulate local immune responses, but systemic responses as well, suggesting that there may be a link between inflammatory processes and their ability to affect the efficacy of chemotherapy by acting on the tumor microenvironment (TME) and initiating an inflammatory response [19]. The TME is normally comprised of a heterogeneous host population of cells such as cancer-associated fibroblasts (CAFs) and tumorassociated macrophages (TAMs) that mediate cancer growth, progression, and reduce the anti-tumor effects of therapies [20].

Recently, a study conducted by Iida et al. reported a correlation between response to anti-cancer therapy and the general function of the gut microbiota [19]. The gut microbiota of mice bearing either EL4 lymphoma, B16 melanoma or MC38 colon cancer was impaired using an antibiotic cocktail prior to tumor cell inoculation, and maintained throughout the study. Once a palpable tumor had formed, a platinum-based chemotherapy or immunotherapy treatment was administered to cohorts with impaired gut bacteria. Analysis of gene expression revealed a downregulation of genes associated with the initiation of an inflammatory response, antigen presentation, adaptive immune response and an upregulation of tissue development, oncogenic and metabolic genes in mice with impaired gut flora. This suggests that an alteration in gut microbiota as mediated through the action of antibiotics can aid in the progression of cancer due to a reduced immune response. Furthermore, the efficacy of platinum-based chemotherapies administered to these mice was also shown to be reduced as evidenced by an increased tumor growth in mice pretreated with antibiotics to impair gut flora. The reduced efficacy of chemotherapy was related to the reduced pro-inflammatory response as a result of impaired gut microbiota suggesting that an inflammatory response is important to the anticancer effects of platinumbased chemotherapy.

Additionally, cancer therapies such as chemotherapeutic agents, alter the composition of the gut microbiota, either directly or indirectly through the activation of an immune response. Due to their abundance and their metabolic activity, 
intestinal bacteria can determine the bioavailability and biological effects of ingested xenobiotics [11]. On the other hand, chemotherapeutic agents have also been shown to have a negative effect on commensal bacteria by altering the composition of the gut microbiota [21]. However, other studies have shown that there is also a compensatory effect whereby gut microbiota is translocated to the intestinal epithelium where it facilitates the activation of dendritic cells and enhances the antitumor effects of CD8+ T-cells [22]. However, the role that gut microbiota play in dampening or enhancing the effectiveness of treatments along with their respective inflammatory response on the progression of cancer is still unknown.

\section{Conclusion}

The relative sum of microorganisms that colonize the human gut is significantly greater than the cells of human origin that comprise the human body, and it plays a crucial role in many vital functions, including structural and metabolic support in the GI tract. Due to its protective role in the maintenance of normal human physiology, disruption of the gut microbiota can promote the generation of an inflammatory environment and can make the host more susceptible to infection, both of which can contribute to cancer development. Following cancer development, the microflora continue to play a role in the absorption and distribution of certain cancer therapies, affecting ttheir therapeutic efficacy. However, the relationship between the gut microbiota and colorectal cancer, does not end here. For example, many cancer therapies affect the gut flora composition, disrupting the homeostatic balance with the host and causing further damage to the host by increasing inflammation and thereby propagating cancer progression. Although there are sufficient data to suggest a cyclic connection between the gut microflora and colorectal cancer particularly through inflammatory pathways, the exact mechanism(s) of action have not yet been elucidated. This emerging field requires additional research in order to utilize the intricate microbiota to increase the efficacy and specificity of cancer therapies, and perhaps better understand oncogenesis as mediated by the presence of gut flora.

\section{Acknowledgements}

This work was supported in part by grants to MR Szewczuk from the Natural Sciences and Engineering Research Council of Canada (NSERC), a private sector cancer funding from the Josefowitz Family, and Encyt Technologies, Inc. to MR Szewczuk.

B Qorri is a recipient of the Queen's Graduate Award (QGA) and the 2017 Terry Fox Research Institute Transdisciplinary Training Program in Cancer Research. M Sambi is a recipient of the QGA. The authors report no other conflicts of interest in this work.

\section{References}

1. Boleij A, Tjalsma H (2012) Gut bacteria in health and disease: a survey on the interface between intestinal microbiology and colorectal cancer.
Biol Rev Camb Philos Soc 87(3): 701-730.

2. O'Hara AM, Shanahan F (2006) The gut flora as a forgotten organ. EMBO Rep 7(7): 688-693.

3. Gagnière J, Raisch J, Veziant J, Barnich N, Bonnet R, et al. (2016) Gut microbiota imbalance and colorectal cancer. World J Gastroenterol 22(2): 501-518.

4. Distrutti E, Monaldi L, Ricci P, Fiorucci S (2016) Gut microbiota role in irritable bowel syndrome: New therapeutic strategies. World Gastroenterol 22(7): 2219-2241.

5. Baker PI, Love DR, Ferguson LR (2009) Role of gut microbiota in Crohn's disease. Expert Rev Gastroenterol Hepatol 3(5): 535-546.

6. Louis P, Hold GL, Flint HJ (2014) The gut microbiota, bacterial metabolites and colorectal cancer. Nat Rev Microbiol 12(10): 661-672.

7. Witsch E, Sela M, Yarden Y (2010) Roles for growth factors in cancer progression. Physiology (Bethesda) 25(2): 85-101.

8. (2002) The links between environmental factors, genetics, and the development of cancer. In: Wilson S JL, Couseens C. Cancer and the environment: Gene-environment interaction. Washington DC: National Academies Press, USA.

9. Uemura $N$ (2002) The magnitude of association between Helicobacter pylori infection and the development of gastric cancer. Scand J Gastroenterol 37(8): 869-870.

10. Munoz N, Castellsague X, De Gonzalez AB, Gissmann L (2006) Chapter 1: HPV in the etiology of human cancer. Vaccine 24 (3): S3/1-S3/10.

11. Zitvogel L, Galluzzi L, Viaud S, Vétizou M, Daillère R, et al. (2015) Cancer and the gut microbiota: An unexpected link. Science translational medicine 7(271): 271.

12. Nistal E, Fernández-Fernández N, Vivas S, Olcoz JL (2015) Factors determining colorectal cancer: The role of the intestinal microbiota. Front Oncol 5: 220.

13. Morrison DJ, Preston T (2016) Formation of short chain fatty acids by the gut microbiota and their impact on human metabolism. Gut Microbes 7(3): 189-200.

14. Valentini M, Piermattei A, Di Sante G, Migliara G, Delogu G, et al. (2014) Immunomodulation by gut microbiota: Role of Toll-like receptor expressed by T cells. Journal of Immunology Research. 2014(2014): 586939.

15. Tjalsma H, Boleij A, Marchesi JR, Dutilh BE (2012) A bacterial driverpassenger model for colorectal cancer: beyond the usual suspects. Nat Rev Microbiol 10(8): 575-582.

16. Gao R, Gao Z, Huang L, Qin H (2017) Gut microbiota and colorectal cancer. Eur J Clin Microbiol Infect Dis 36(5): 757-769.

17. Vetizou M, Pitt JM, Daillere R, Lepage P, Waldschmitt N, et al. (2015) Anticancer immunotherapy by CTLA-4 blockade relies on the gut microbiota. Science 350(6264): 1079-1084.

18. Sivan A, Corrales L, Hubert N, Williams JB, Aquino-Michaels K, et al. (2015) Commensal Bifidobacterium promotes antitumor immunity and facilitates anti-PD-L1 efficacy. Science. 350(6264): 1084-1089.

19. Iida N, Dzutsev A, Stewart CA, Smith L, Bouladoux N, et al. (2013) Commensal bacteria control cancer response to therapy by modulating the tumor microenvironment. Science 342(6161): 967-970.

20. Grivennikov SI, Greten FR, Karin M (2010) Immunity, inflammation, and cancer. Cell 140(6): 883-899.

21. Touchefeu Y, Montassier E, Nieman K, Gastinne T, Potel G, et al. (2014) Systematic review: the role of the gut microbiota in chemotherapy- or radiation-induced gastrointestinal mucositis - current evidence and potential clinical applications. Aliment Pharmacol Ther 40(5): 409421. 
22. Paulos CM, Wrzesinski C, Kaiser A, Hinrichs CS, Chieppa M, et al. (2007) Microbial translocation augments the function of adoptively transferred self/tumor-specific CD8+ T cells via TLR4 signaling. J Clin Invest 117(8): 2197-2204.

\section{Your next submission with JuniperPublishers will reach you the below assets}

- Quality Editorial service

- Swift Peer Review

- Reprints availability

- E-prints Service

- Manuscript Podcast for convenient understanding

- Global attainment for your research

- Manuscript accessibility in different formats

( Pdf, E-pub, Full Text, audio)

- Unceasing customer service

Track the below URL for one-step submission https://juniperpublishers.com/online-submission.php 